\title{
Netrin-1 interacts with amyloid precursor protein and regulates amyloid- $\beta$ production
}

\author{
FC Lourenço ${ }^{1}$, V Galvan ${ }^{2,4}$, J Fombonne ${ }^{1,4}$, V Corset ${ }^{1,4}$, F Llambi ${ }^{1}$, U Müller ${ }^{3}$, DE Bredesen ${ }^{2,5}$ and P Mehlen ${ }^{\star 1,2,5}$
}

The $\beta$-amyloid precursor protein (APP) is an orphan transmembrane receptor whose physiological role is largely unknown. APP is cleaved by proteases generating amyloid- $\beta(A B)$ peptide, the main component of the amyloid plaques that are associated with Alzheimer's disease. Here, we show that APP binds netrin-1, a multifunctional guidance and trophic factor. Netrin-1 binding modulates APP signaling triggering APP intracellular domain (AICD)-dependent gene transcription. Furthermore, netrin-1 binding suppresses $A \beta$ peptide production in brain slices from Alzheimer model transgenic mice. In this mouse model, decreased netrin-1 expression is associated with increased $A \beta$ concentration, thus supporting netrin- 1 as a key regulator of $A \beta$ production. Finally, we show that netrin-1 brain administration in Alzheimer model transgenic mice may be associated with an amelioration of the Alzheimer's phenotype.

Cell Death and Differentiation (2009) 16, 655-663; doi:10.1038/cdd.2008.191; published online 16 January 2009

Alzheimer's disease (AD), the most common form of dementia, is a progressive neurodegenerative disorder characterized by extracellular deposits of $A \beta$ peptide in senile plaques, intraneuronal neurofibrillary tangles, synapse loss, and cognitive decline. ${ }^{1}$ It is widely believed that the accumulation of $\mathrm{A} \beta$, a small peptide with a high propensity to form oligomers and aggregates, is central to the pathogenesis of $A D$. $A \beta$ derives from the proteolytic cleavage of the transmembrane protein, APP. ${ }^{2}$ Although a considerable amount is known about interacting proteins and processing events for APP, the physiological role(s) of APP and its related family members, APLP1 and APLP2 (amyloid precursor-like proteins 1 and 2), is (are) still poorly understood. ${ }^{2,3}$ APP has been proposed to function in cell adhesion and motility, as well as synaptic transmission and plasticity (for a review, see Turner et al. ${ }^{3}$ ).

The cloning and characterization of APP revealed that it possesses many features reminiscent of a membraneanchored receptor. However, to date, no clear candidate has emerged as the major ligand triggering APP-mediated signal transduction (although several molecules have been shown to bind APP, such as collagen (types I and IV), heparan sulfate proteoglycan, laminin, glypican, and more recently TAG-1 $)^{4-7}$ - at least in part because the signal transduction mediated by APP remains incompletely understood. Here, we show that netrin-1 functions as a ligand for APP, it modulates APP signaling, and we also show that it regulates $\mathrm{A} \beta$ peptide production in Alzheimer model transgenic mice.

Netrin-1 is a soluble molecule initially described by TessierLavigne and colleagues ${ }^{8}$ as a chemotropic cue involved in axon guidance. Netrin-1 plays a critical role during nervous system development by mediating chemoattraction of axons and neurons through its interaction with DCC (deleted in colorectal cancer). ${ }^{8-10}$ However, netrin-1 has also been described as a survival factor, involved in, among other effects, the regulation of tumorigenesis. ${ }^{11}$ Such dual effects on nervous system development and tumorigenesis are characteristics of the so-called dependence receptors, of which DCC represents an example. ${ }^{12}$

While performing a mass spectrometric analysis to identify proteins interacting with either DCC or with the DCC/netrin-1 complex, we sequenced APLP1 as a candidate coimmunoprecipitant with the DCC/netrin-1 complex (Supplementary Figure 1). This led to the question of whether netrin-1 may actually interact with APP family members. We show here that APP is a functional receptor for netrin-1. We also show that netrin- 1 negatively regulates the $A \beta$ level in adult brain and that netrin-1 brain administration may represent an appealing strategy to improve the Alzheimer's phenotype.

\section{Results and Discussion}

Netrin-1 interacts with APP. APP or APLP-1 and netrin-1 were coexpressed in HEK293T cells and, as shown in Figure 1a and Supplementary Figure 2ab, APLP1 and APP coimmunoprecipitated with netrin-1. As a negative control, another transmembrane receptor, TrkC, failed to coimmunoprecipitate with netrin-1 (Figure 1a). Similar results were obtained when netrin-1 and APP were coexpressed in

\footnotetext{
${ }^{1}$ Apoptosis, Cancer and Development Laboratory - Equipe labellisée 'La Ligue', CNRS UMR5238, Centre Léon Bérard, University of Lyon., 69008 Lyon, France; ${ }^{2}$ The Buck Institute for Age Research, Novato, CA 94945, USA and ${ }^{3}$ Institute for Pharmacy and Molecular Biotechnology (IPMB), University of Heidelberg, Germany ${ }^{*}$ Corresponding author: P Mehlen, Apoptosis, Cancer and Development Laboratory- Equipe labellisée 'La Ligue', CNRS UMR5238, University of Lyon, Centre Léon Bérard-CNRS UMR5238, 28 rue Laennec, Lyon, 69008, France. Tel: + 33478782 870; Fax: + 33478782 887; E-mail: mehlen@lyon.fnclcc.fr

${ }^{4}$ These authors contributed equally to this work.

${ }^{5}$ These two authors are co-senior authors.

Keywords: Alzheimer's disease; netrin-1; amyloid- $\beta$; amyloid precursor protein

Abbreviations: $\mathrm{AD}$, Alzheimer's disease; $\mathrm{A} \beta$, amyloid- $\beta$; APP, amyloid precursor protein; $\mathrm{DCC}$, deleted in colorectal cancer; APLP1, amyloid precursor-like protein 1; FGF, fibroblast growth factor

Received 10.7.08; revised 14.11.08; accepted 25.11.08; Edited by M Deshmukh; published online 16.1.09
} 
B103 cells (data not shown). Not only ectopically expressed APP (Figure 1a) but also endogenous APP could be immunoprecipitated with netrin-1 in HEK293T cells
(Figure 1b). We next analyzed whether netrin-1 could be recruited to the plasma membrane of APP-expressing B103 cells. As shown in Figure 1c, although netrin-1 failed to a

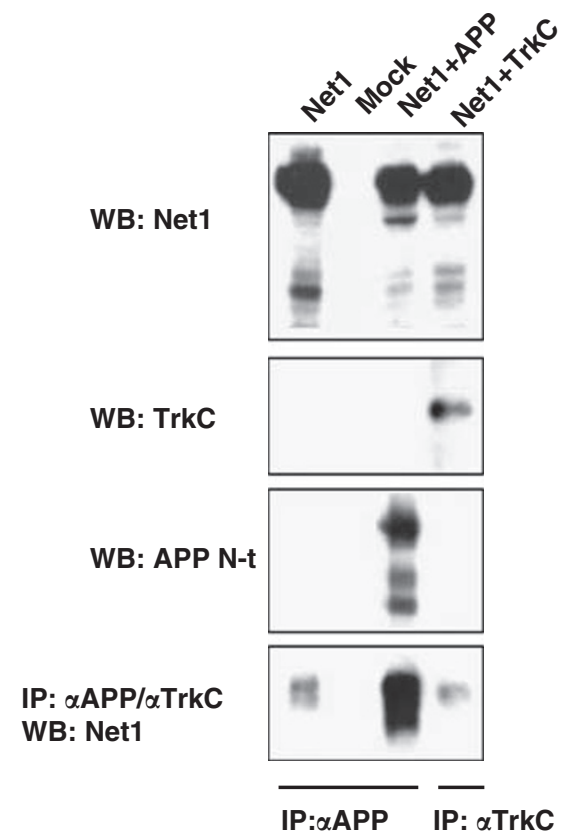

d
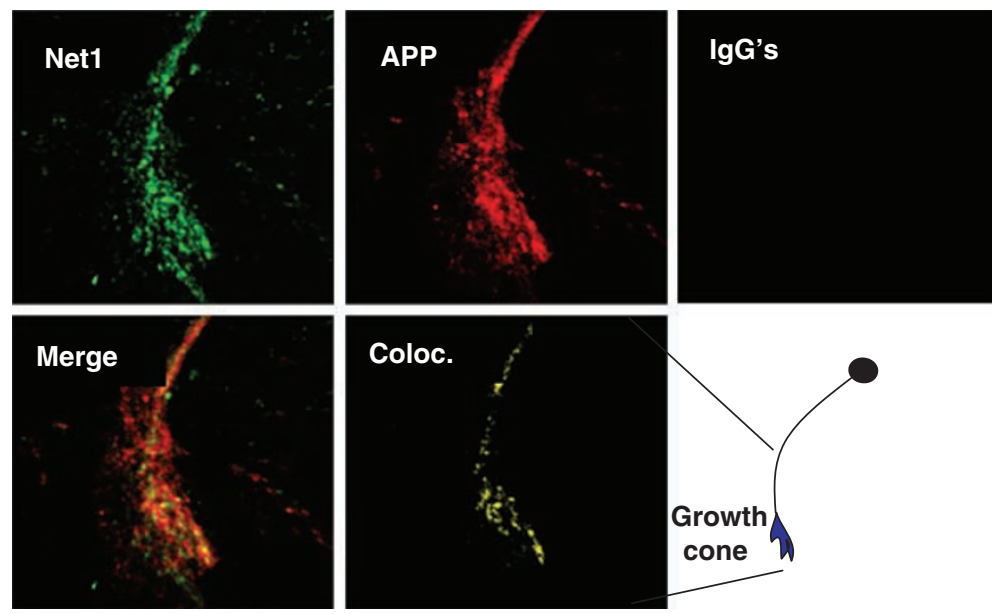

$\mathbf{e}$

IP: ac-myc

WB: APP C-t
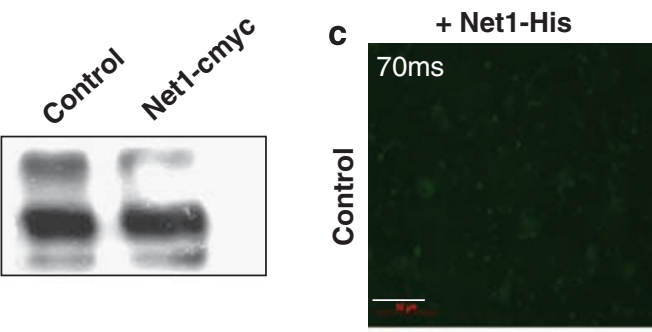

WB: c-myc

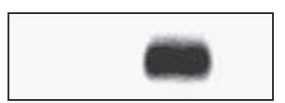

$\frac{0}{4}$

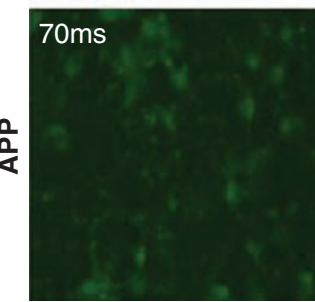

\section{Coloc.}
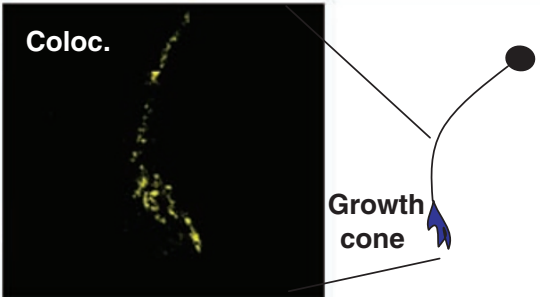

WB: APP N-t

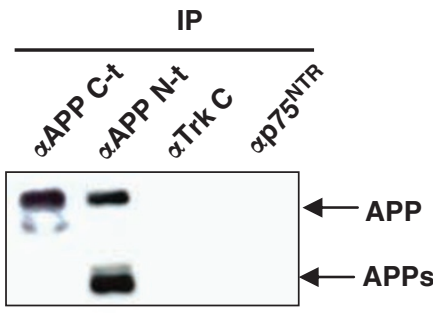

f

IP: $\alpha$ APP

WB : Net-1

WB : APP

WB : Net-1
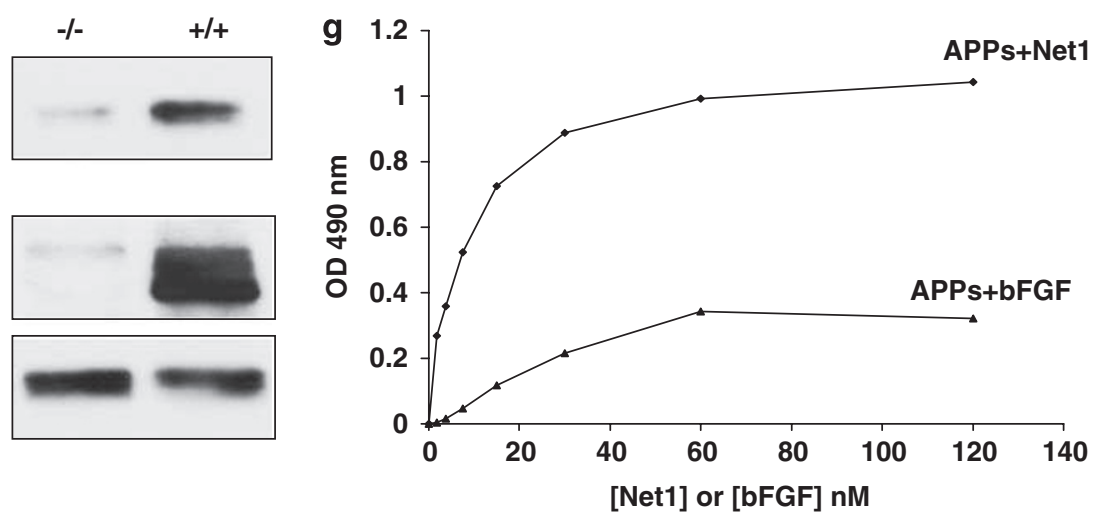
show affinity for control-transfected $\mathrm{B} 103$ cells, it was recruited to APP-transfected $\mathrm{B} 103$ cells. Because these assays were performed in cells in which APP or netrin-1 were expressed in a nonphysiological setting, we investigated the putative APP/netrin-1 interaction in primary cortical neurons from E16.5 mouse embryos. We first analyzed whether endogenous APP is colocalized with endogenously expressed netrin-1 by confocal microscopy. As shown in Figure 1d, APP colocalizes with netrin-1, especially in growth cones of these cortical neurons. We then performed the coimmunoprecipitation of endogenous proteins. As shown in Figure 1e, netrin-1 interacts with APP in the developing cortex, whereas in the same setting, netrin-1 fails to interact with $\mathrm{p} 75^{\mathrm{NTR}}$. Moreover, in APP mutant developing brains, netrin-1 is not pulled down after APP immunoprecipitation (Figure 1f). Thus, endogenous netrin-1 specifically interacts with endogenous APP in the developing brain.

To further analyze whether APP interaction is restricted to netrin-1, we performed immunoprecipitation using different netrin family members. In addition to netrin-1, netrin- 2 was also found to interact with APP, whereas we failed to detect the interaction of APP with the more divergent netrin molecule, netrin G1 (Supplementary Figure 2c). Because both netrins and APP are heparin-binding proteins, ${ }^{10,13}$ we assessed whether a netrin-1 mutant deleted for domain $C$ (which contains the major heparin-binding domain, but is dispensable for netrin-1 function ${ }^{10}$ ) retains the ability to interact with APP. As shown in Supplementary Figure 2c, this mutant netrin-1 does indeed retain the ability to interact with APP.

To exclude the possibility of an indirect interaction between netrin-1 and APP, direct in vitro interaction was assessed by immunoprecipitation and ELISA assays on recombinant $\alpha$ APPs or DCC ectodomain, with recombinant netrin-1 or bFGF, a molecule that shares many characteristics of netrin-1. ${ }^{14}$ As shown in Figure $1 \mathrm{~g}$ and Supplementary Figure 2d, immunoblots and ELISA assays revealed a specific interaction of APP with netrin-1, whereas bFGF/APP failed to show specific binding. The affinity of netrin-1 for APPs is the same order of magnitude as its affinity for DCC (estimated $\mathrm{Kd}_{\mathrm{APP} / \text { netrin }}$ of $6 \mathrm{nM}$, compared to the known $\mathrm{Kd}_{\mathrm{DCC} / \text { netrin-1 }}$ of $\left.10 \mathrm{nM}^{10}\right)$. Taken together, these data support the notion that netrin-1 interacts with APP with an affinity that is similar to that of its previously described physiological interaction with DCC.

We next attempted to define the APP domain required for the APP/netrin-1 interaction. The carboxy-terminal C83 derived from the $\gamma$-secretase cleavage of APP fails to interact with netrin-1, hence further showing that netrin-1 interacts with APP ectodomain (Figures $2 \mathrm{a}$ and $\mathrm{b}$ ). Interestingly, the C100 protein, derived from the $\beta$-secretase cleavage of APP (see scheme in Figure 2a), is pulled down with netrin-1 (Figure $2 b$ ). Taken together with the finding that, in vitro, the $\alpha$ APPs protein interacts with netrin-1 (Figure 1j and Supplementary Figure 2b), these observations suggested that a binding region of APP is localized between the $\beta$-cleavage and the $\alpha$-cleavage sites - that is, the 17 aminoterminal residues of $\mathrm{A} \beta$, even though this may not be the only interacting domain of APP with netrin-1. Along this line, recombinant netrin-1 interacts in a concentration-dependent manner with the $\mathrm{A} \beta$ peptide (Figure $2 \mathrm{c}$ ). Thus, $\mathrm{A} \beta$ appears to interact with netrin-1 (although we cannot exclude the possibility that this interaction is biologically irrelevant and only because of the welldescribed "sticky" nature of the $\mathrm{A} \beta$ peptide). Interestingly, not only $\mathrm{A} \beta$ but also a smaller fragment of $\mathrm{A} \beta, \mathrm{A} \beta 1-17$ - that is, the 17 first amino acids of $A \beta$ (a less toxic peptide than full-length $\mathrm{A} \beta$ ) - interacted with netrin-1, although with a reduced affinity $\left(\mathrm{Kd}_{\mathrm{A} \beta / \text { netrin-1 }}: 22 \mathrm{nM}, \mathrm{Kd}_{\mathrm{A} \beta 1-17 / \text { netrin-1 }}\right.$ : $30 \mathrm{nM}$; Figure 2d). Thus, the APP ectodomain interacts with netrin-1 at least in part in a region that includes the $A \beta 1-17$ region of APP

Netrin-1 affects APP signaling. We next investigated whether netrin-1 may modulate the downstream signaling of APP. The adaptor protein DAB-1 has been shown to interact with the intracellular domain of APP. ${ }^{15}$ As shown in Supplementary Figure $3 a$, netrin-1 enhanced the recruitment of DAB-1 to APP. Similarly, the adaptor protein Fe65 has been shown to interact with the intracellular domain of APP and to provide a mechanism for the coupling of APP to the cytoskeleton. ${ }^{16} \mathrm{Fe} 65$ has also been shown to be responsible for APP intracellular domain (AICD)-dependent gene transcription. Indeed, even though it is still a matter of debate, it is believed that in the presence of Fe65, the intracellular domain of APP migrates to the nucleus and initiates APP-dependent transcription of a specific set of

Figure 1 Netrin-1 interacts with APP. (a) HEK293T cells were transiently transfected with myc-tagged netrin-1 and/or APP and/or TrkC. Cell lysate was utilized for immunoprecipitation, using either an anti-N-terminal APP antibody or an anti-TrkC antibody. Immunoblots were probed with antibodies raised against N-terminal APP, netrin-1, or TrkC. (b) HEK293T cells were transfected with netrin-1-expressing construct or not, and endogenous APP was, after c-myc (netrin-1) pull-down, revealed using an anti-Cterminal APP antibody. (c) B103 cells were transfected with mock- or APP-expressing plasmid and treated for $1 \mathrm{~h}$ with his-netrin-1. Anti-HIS immunohistochemistry is shown. (d) Colocalization of 5A3/1G7 (APP extracellular domain) with netrin-1 in growth cones of primary cortical neurons. Primary cultures of neurons from DBA/2J embryos were fixed in 4\% PFA and stained with 5A3/1G7 and antinetrin 64 or with mouse and rabbit lgGs followed by Alexa568- and Alexa488-conjugated antimouse and antirabbit secondary antibodies, respectively. Stacks of images $(z$-step $=0.25 \mu \mathrm{m})$ were acquired with a laser scanning confocal microscope. Analysis of colocalization was performed using the Coloc algorithm in Imaris Bitplane. The Pearson correlation coefficient of channels A (green) and B (red) inside the colocalized region was used as a measure of the degree of colocalization. ${ }^{36}$ Panels shown are Net1 (netrin-1), APP, merge, Coloc. (colocalization channel), and IgGs (mouse and rabbit IgGs). A cartoon of the region of a cortical neuron shown in the various panels is indicated. (e) Cortexes from E16.5 mouse embryos were collected and semidissociated, and cells lysates were submitted to immunoprecipitation using anti-APP (C-terminal or N-terminal), anti-TrkC or anti-p $75^{\mathrm{NTR}}$ antibody for the pull-down. Immunoblot were then performed using either APP, p75 $5^{\text {tr }}$ or TrkC antibody. (f) Immunoprecipitations were performed with a mixed mouse monoclonal anti-APP (5A3/1G7) antibody from E16.5 mouse brain of either wild-type embryo $(+/+)$ and APP mutant $(-/-)$. Netrin-1 and APP immunoblot using a monoclonal antinetrin-1 (dilution 1/1000) (R\&D system) and monoclonal anti-APP (5A3/1G7) antibodies are shown. (g) An Elisa assay was developed to determine the $\mathrm{Kd}_{\mathrm{APP} / \text { netrin. }} . \mathrm{In}$ all, $2.5 \mu \mathrm{g} / \mathrm{ml}$ of $\alpha \mathrm{APPs}$ protein was coated in 96-wells plate and various netrin-1 concentrations were added. Similar experiment was performed using the pair APP/bFGF. Quantification of the interaction is indicated here by the measurement of the optic density (intensity). Determination of Kd was derived from a simulated Scatchard plot (bound/estimated free $=f($ bound)) 


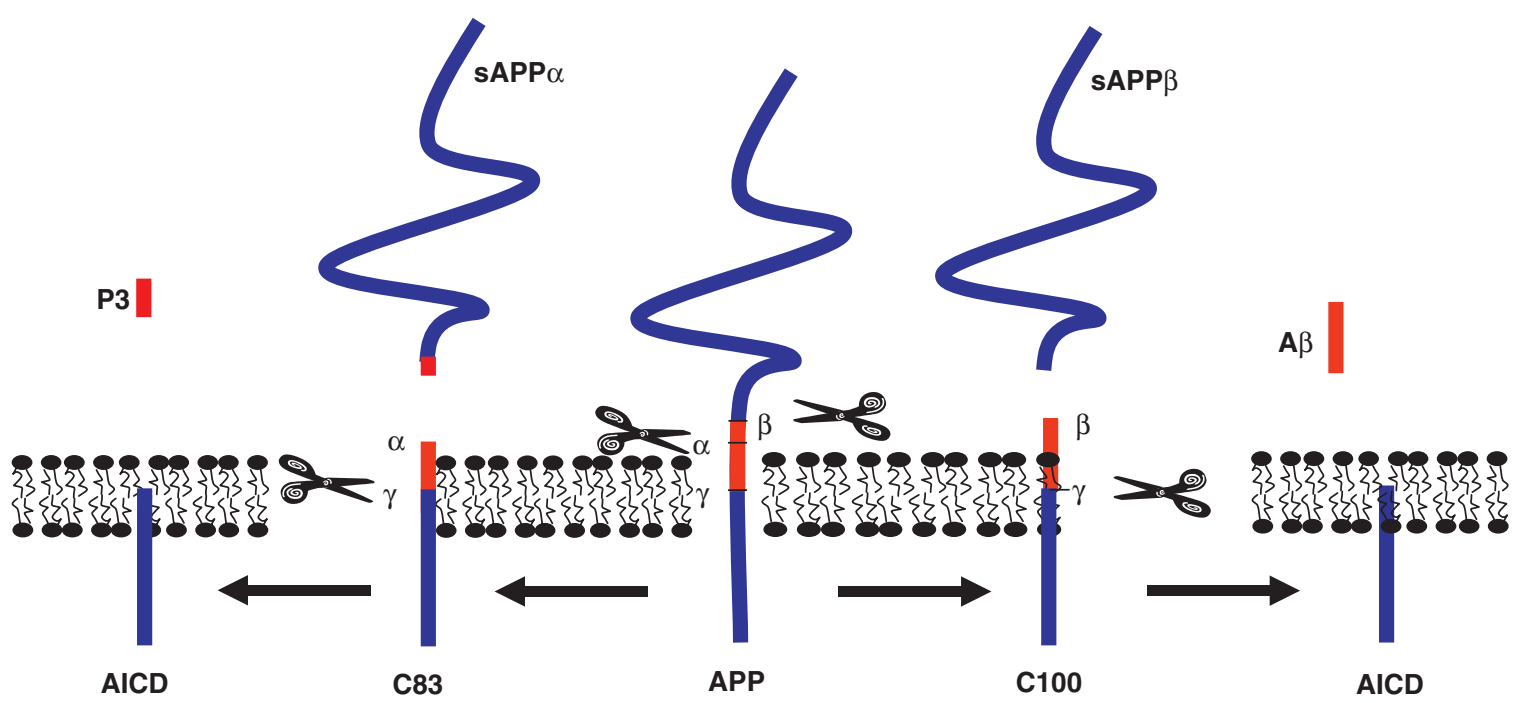

b

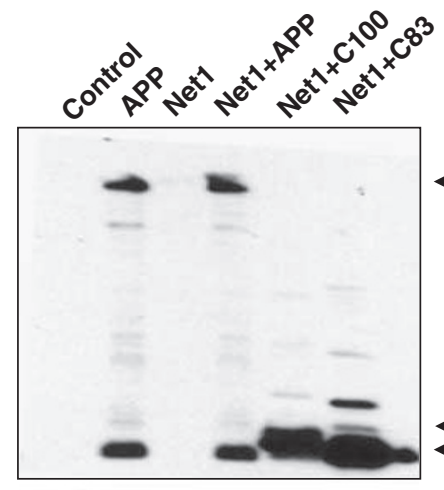

WB:APP C-t

WB: Net1

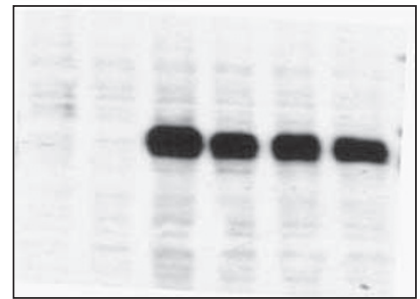

IP: $\alpha$ APP C-t WB: Net1

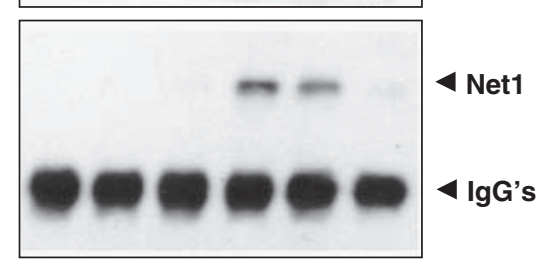

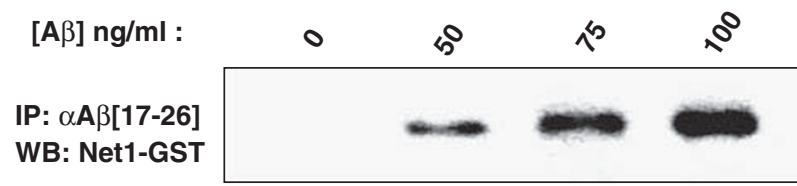
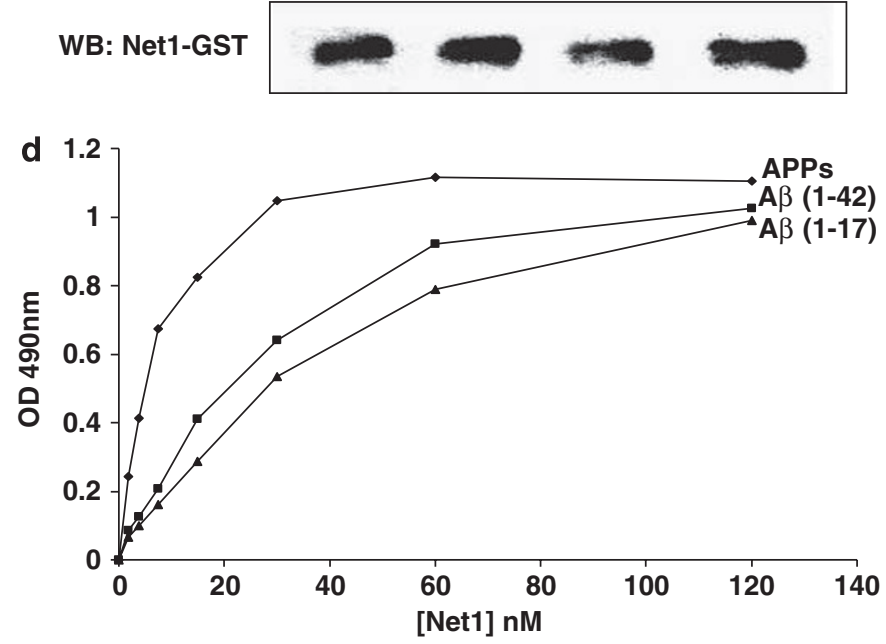

Figure 2 A netrin-1-binding domain lies within the A $\beta$ region of APP. (a) Schematic representation of APP. (b) HEK293T cells were cotransfected with a C100 (APP597695) or C83 (APP613-695)-expressing construct in the presence or in the absence of a netrin-1 expression construct. Immunoprecipitation was performed using anti-APP (C-terminal) for the pull-down and an antibody raised against netrin-1 or against the C-terminal domain of APP for the immunoblot. (c) Netrin-1 immunoprecipitates with A $\beta$. In all, $150 \mathrm{ng}$ of GST/netrin-1 were incubated with increasing concentrations of $A \beta$ in $1 \mathrm{ml}$ of reaction buffer. Pull-down was performed using anti-A $\beta$-specific antibody, and netrin1 was detected by western blot using anti-GST antibody. (d) An ELISA assay was performed as in Figure $1 \mathrm{~g} ; 0.18 \mu \mathrm{g} / \mathrm{ml}$ of A $\beta$ or $0.07 \mu \mathrm{g} / \mathrm{ml}$ of $\mathrm{A} \beta 1-17 \mathrm{protein}$ was coated in 96-wells plate and various netrin-1 concentrations were added. Quantification of the interaction is indicated here by the measurement of the optic density (intensity)

genes through interaction with TIP60. ${ }^{17-20}$ Figure 3a shows that, in APP-transfected B103 cells, the Fe65 interaction with APP is mainly observed when netrin-1 is added. According to the model proposed, this netrin-1-dependent enhanced interaction of Fe65 with APP could lead to an increased AICD activity. To test this, we first used the transactivation assay developed previously ${ }^{17}$ in which the DNA-binding domain of Gal4 is fused to APP, and transactivation is monitored with a Gal4-dependent reporter plasmid. As shown in Figure 3b, netrin-1 triggers APP-dependent gene transactivation in HEK293T, whereas bFGF has no effect on the Gal4-dependent reporter. 
a

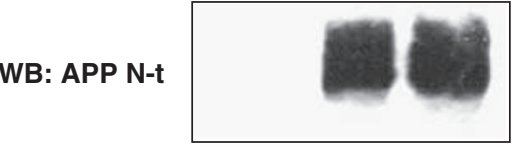

WB: Fe65

(endogen)

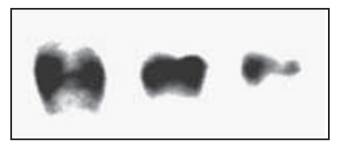

IP: $\alpha \mathrm{Fe} 65$

WB: APP N-t

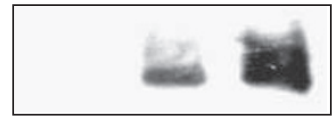

b

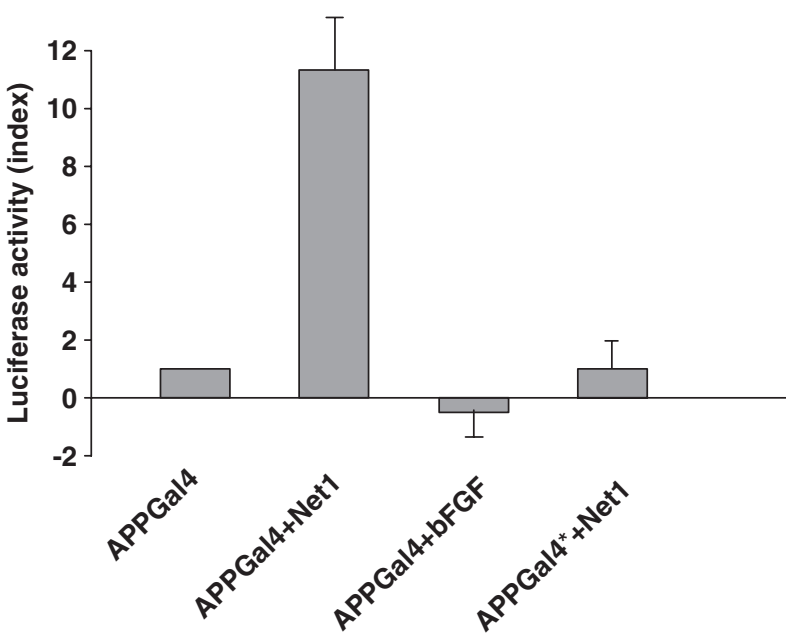

anti-APP C-t+Toto-3

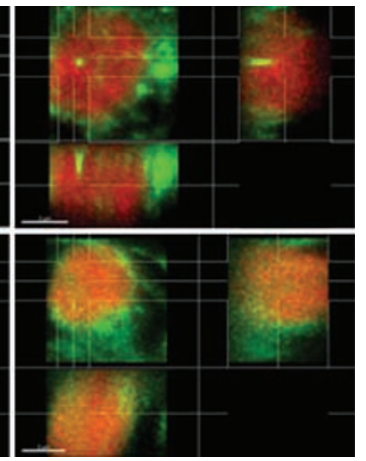

d

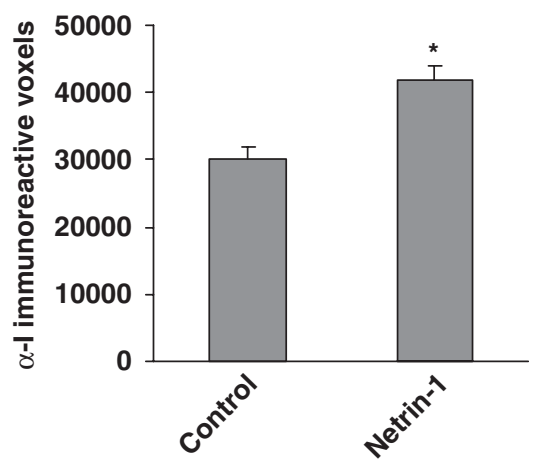

Figure 3 Netrin-1 activates APP signaling. (a) B103 cells were transiently transfected with APP and further incubated with (or without) netrin-1. Endogenous Fe65 was immunoprecipitated using anti-Fe65 antibody, and N-terminal anti-APP antibody was used to detect APP within the pull-down. The upper panels represent APP and Fe65 before the pull-down, the lower panel being APP detected in the immunoprecipitation. (b) HEK293T cells were cotransfected with APP-Gal4 or APP*-Gal4 together with a Gal4-luciferase reporters (pG5E1B-luc) construct in a 1:1 ratio. In all, $300 \mathrm{ng} / \mathrm{ml}$ of netrin-1 (or of bFGF) was added to the culture after 24 and $48 \mathrm{~h}$ after transfection; cells were collected and cell lysates were assessed for luciferase activity using the Promega luminescence measurement assay and a Victor biostation (Perkin-Almer). A histogram plot is presented. Standard deviations are indicated $(n=5)$. (c and d) Primary neuronal cultures from E16.5 hAPP transgenic embryos (PDAPP(J20) in C57BL/6J background) were treated with vehicle (PBS) or with $300 \mathrm{ng} / \mathrm{ml}$ netrin-1 added to the culture media every $24 \mathrm{~h}$ for 3 days, starting 1.5 day after plating. Cultures were fixed, treated with RNAse, and stained with a 1:1000 dilution of an antibody specific for the C-terminal domain of APP (amino acids 649-664, antiserum I (R1155) ${ }^{37}$ ) followed by Alexa488-conjugated donkey antirabbit IgG (Invitrogen) and counterstained with TOTO-3 to visualize DNA. Stacks of images $(z$-step $=250 \mathrm{~nm})$ were acquired with a laser scanning confocal microscope (Nikon PCM-2000) at $\times 600$ magnification and collected with SimplePCI (Compix Inc., Sewickley, PA, USA) software. For each condition, five separate fields were chosen in which individual cells were clearly distinguishable (avoiding clumps of neuronal bodies). A representative maximum intensity projection image of fields acquired for each condition is shown in Supplementary Figure $3 \mathrm{~b}$. Distribution of intensity of anti-I immunoreactivity across nuclei is shown in Supplementary Figure 3c. (c and d) Individual volumes $(9 \times 9 \times 6 \mu \mathrm{m})$ of single nuclei (control, $n=53$; netrin-1, $n=51$ ) were cropped from stacks of confocal images and analyzed separately using the Imaris Isosurface algorithm (Imaris Bitplane, Zurich, Switzerland). (c) Maximum intensity projections of stacks of control and netrin-1-treated neuronal nuclei. Representative images are shown. (d) Numbers of anti-I immunoreactive voxels in neuronal nuclei. A significant increase of APP C-terminal immunoreactive voxels was observed in nuclei of neurons and had been treated with netrin-1 $(P<0.05$, unpaired Student's $t$-test $)$

This netrin-1-dependent, APP-dependent transactivation is abolished when APP is mutated at the Fe65 binding site. To further study netrin-1 effects on AICD, AICD was analyzed in primary cortical neurons derived from E16.5 embryos from transgenic mice expressing a human APP minigene carrying the Swedish and Indiana familial AD mutations (PDAPP). ${ }^{21}$ As AICD-nuclear localization is an important - even though dispensable - event in AICD-dependent transactivation, ${ }^{19}$ we assessed the effect of netrin-1 on the translocation of APP C-terminal-immunoreactive fragments into nuclei of
hAPP transgenic cortical neurons. As shown in Figure $3 \mathrm{c}$ and $\mathrm{d}$ and Supplementary Figure 3bc, APP C-terminalimmunoreactive fragments are significantly increased in nuclei of netrin-1-treated neurons. We next determined whether KAl1, a gene whose expression has been shown to be AICD-dependent in different cell systems, ${ }^{22}$ is induced in response to netrin-1. Therefore, quantitative RT-PCR on KAl1 was performed on RNA extracted from netrin-1treated versus nontreated primary cortical neurons. Netrin-1 induced an increase in KAl1 expression (fold induction: 
$4.3 \pm 1.2)$. Thus, even though we cannot exclude the possibility that netrin-1-dependent KAl1 expression is due to a netrin-1 receptor other than APP, these observations, taken together with the transactivation assay performed in HEK293T cells, strongly support the notion that netrin-1 triggers AICD-dependent gene transactivation. Taken together, these results suggest that netrin-1 is likely to exert a functional effect on APP signaling.

Netrin-1 controls $A \beta$ levels in an AD transgenic mouse model. Because AD pathogenesis is thought to be mediated at least in part through APP processing resulting in $A \beta$ production, we next evaluated the effect of netrin-1 on $\mathrm{A} \beta$ production in hAPP transgenic mice that model AD (PDAPP). ${ }^{21}$ Whole brain slice cultures from PDAPP transgenic and nontransgenic littermates were treated with vehicle or with netrin-1 and were evaluated by ELISA assay for the production of $\mathrm{A} \beta 1-40$ and $\mathrm{A} \beta 1-42$. As shown in Figure $4 \mathrm{a}$, the Alzheimer model transgenic mice displayed a marked increase in $\mathrm{A} \beta 1-40$ and $\mathrm{A} \beta 1-42$ net productions over that of the control mice (nontransgenic littermates), but this was suppressed by the addition of netrin-1. To assess the role of netrin-1 in a complementary, loss-of-function experiment, we crossed netrin-1 hemizygous mice (netrin nulls are nonviable) with hAPP transgenic mice, and then quantitated cerebral $\mathrm{A} \beta$ concentrations in the progeny by ELISA assay. The PDAPP transgenic netrin-1 hemizygotes (PDAPP/netrin-1 +/-) showed a decreased netrin-1 level in the cortex compared to wild-type mice (PDAPP/netrin-1 + / + ; Figure $4 \mathrm{~b}$, inset). Quantitation of $A \beta$ revealed a significant increase in $A \beta$ levels in netrin-1 hemizygotes compared to wild-type mice (Figure $4 b$ ), hence strengthening the view of netrin-1 as a key regulator of $\mathrm{A} \beta$ level. Interestingly, netrin-1 is expressed not only during brain development but also in adult brain. Some brain regions still express high levels of netrin-1, as for example the striatum, the ventral tegmentum, and the substantia nigra (Figure $4 \mathrm{c}$ and $\mathrm{d}$ and Livesey and Hunt $^{23}$ ). Although these brain regions are not those most commonly associated with pathology in $A D$, these regions have indeed been shown to be affected in Alzheimer's. Furthermore, netrin-1 expression is also detected in the main regions of $\mathrm{A} \beta$ deposition and Alzheimer-related pathology including hippocampus and dentate gyrus (Figure 4c and d and Colicos ${ }^{24}$ ). It will, therefore, be of interest to determine whether the concentration ratio of $\mathrm{A} \beta$ peptides to netrin- 1 in the adult brain represents a critical determinant of the development of $A D$.

Initial studies toward the use of netrin-1 or derivatives to ameliorate the Alzheimer's phenotype. Netrin-1 thus shows multiple effects that make it a potential candidate for therapy in AD: (i) it markedly decreases $A \beta 1-40$ and $A \beta 1-42$ concentrations in brain slices from Alzheimer model transgenic mice; (ii) it interacts with $\mathrm{A} \beta$ peptide, with as-yet-unknown effects on $\mathrm{A} \beta$ oligomerization and clearance; (iii) it displays a neurotrophic effect. ${ }^{25}$ As a first step toward a potential therapeutic use of netrin-1 in $A D$ model transgenic mice, we delivered netrin-1 through the intracerebroventricular pump injection in PDAPP mice. As shown in Figure 4e, a 13-day netrin-1 brain injection (but not vehicle injection) was associated with a decreased $\mathrm{A} \beta$ level. Furthermore, consistent with the observed reduction in $A \beta$ levels in netrin1-treated Alzheimer's mice, preliminary results suggest that PDAPP mice infused with netrin-1 showed significantly improved memory function. Indeed, as reported previously, ${ }^{26,27}$ PDAPP mice lacked the normal preference for novel stimuli, a trend that was exacerbated in the olfactory novelty (ON) recognition tests (Figure 4f). In contrast, preliminary results show that netrin-1-infused PDAPP mice have a pronounced increase in their preference for the exploration of novel stimuli (Figure 4f). Even though we cannot exclude the possibility that this netrin-1-dependent memory enhancement was mediated by a receptor other than APP, this observation strengthens the rationale for mimicking netrin-1 as a putative treatment in AD. Thus, although further work will be required to prove that the delivery of netrin-1, a netrin-1 domain, or a netrin-1 mimetic may be of interest for the amelioration of $A D$ pathology in this transgenic mouse model, the observations that netrin-1 acts as a ligand for APP, controls $\mathrm{A} \beta$ level, and may positively impact memory function, opens a novel avenue of research and potential application that links netrin-1, APP-mediated signal transduction, A $\beta$ synthesis, neurite extension/retraction, and AD.

\footnotetext{
Figure 4 Netrin-1 regulates net A $\beta$ peptide level. (a) Brain slices from PDAPP transgenic mice and control nontransgenic littermates (N-PDAPP) were cultured in the presence or absence of netrin-1 (1.5 nM). Supernatants were harvested after $3-5$ days and evaluated by ELISA assay for $A \beta 1-40$ and $A \beta 1-42$. In all, $90 \mathrm{ng} / \mathrm{ml}$ of netrin-1 were added to all $\mathrm{A} \beta$ standards to rule out netrin-1 interference with binding of the antibodies used in the ELISA to their epitopes on A $\beta$. NGF (250 ng/ml) or IGF-1 (100 ng/ml) was also added as control and failed to have any effect on A $\beta$ level (not shown). (b) Netrin-1 expression (inset) and net A $\beta$ production were measured in 5-7-month old PDAPP netrin-1 $+/$ - or PDAPP/netrin-1 $+/+$ mice by ELISA. Fold increase is presented as the ratio between average A $\beta$ levels detected in PDAPP/netrin- $1+/-$ mice and that in PDAPP/netrin-1 $+I+$ mice. Four cohorts of the animals of similar age (netrin- $1+I+$ and $+/-$ ) were studied. Total number of mice studied were 16 . ANOVA test was used comparing $+I-$ versus $+I+$ in the four groups $(P<0.027)$, comparing $+I-$ versus $+I+$ in the whole population $(P=0.0005)$. (c) Netrin-1 immunoblot in mouse nervous system. Cortex (Ctx), cerebellum (Cb), spinal cord (ASC), striatum (Str), and hippocampus (Hi) from adult mice or as control E13 mice embryonic spinal cord (ESC) were dissected out, and immunoblots using antinetrin-1 or anti- $\beta$-actin (as a loading control) antibody are shown. (d) Netrin-1 expression in adult brain followed by LacZ activity in netrin-1 $+/$ - mutant mice. Control: Xgal staining on net $+I+$ brain mouse. Cortex (Ctx), cerebellum (Cb), striatum (Str), and hippocampus (Hi) are indicated. Netrin-1 transcript in adult brain was followed using the analysis of transgenic mouse in which the netrin-1 promoter drives the expression of LacZ. ${ }^{9}$ Brains of 8 months old netrin-1 mutant mice net $+I$ - or net $+I+$ in the NPDAPP genetic background were excised and incubated overnight with a $1.3 \mathrm{mg} / \mathrm{ml} \mathrm{X-Gal} \mathrm{solution,} \mathrm{then} \mathrm{fixed} \mathrm{for} 30 \mathrm{~min}$ at room temperature with PFA 4\% and included in $3 \%$ low melting agarose (Cambrex). Brain sections (200 $\mu \mathrm{m}$ ) were performed using a vibratome and incubated for 2 days with $1.3 \mathrm{mg} / \mathrm{ml} \mathrm{X}$-Gal solution (Euromedex) at $4^{\circ} \mathrm{C}$. (e and f) 6-8 months PDAPP transgenic mice $(n=16)$ were infused intracerebrally with $100 \mu$ of artificial cerebrospinal fluid (saline) with or without $130 \mu \mathrm{g} / \mathrm{ml}$ recombinant mouse netrin-1 for 12-13 days using Alzet osmotic pumps. (e) A $\beta-40$ and A $\beta 1-42$ levels measured by ELISA as in panel b. (f) preference for novel stimuli in the ON test as described in the Materials and Methods section
} 


\section{Materials and Methods}

Cells, transfection procedures, and purified and recombinant proteins. Transfections of HEK293T (human embryonic kidney) or B103 (neuroblastoma) cells were performed using the lipofectamine reagent
(Invitrogen, Carlsbad, CA, USA). Primary cortical neurons were obtained from E16.5 embryos and cultured in neurobasal media supplemented with B27 (Invitrogen). Recombinant Flag-netrin-1 was from Apotech Corp (Axxora). C-myctagged netrin-1 was purified from netrin-1-producing 293-EBNA cells as before. ${ }^{8}$
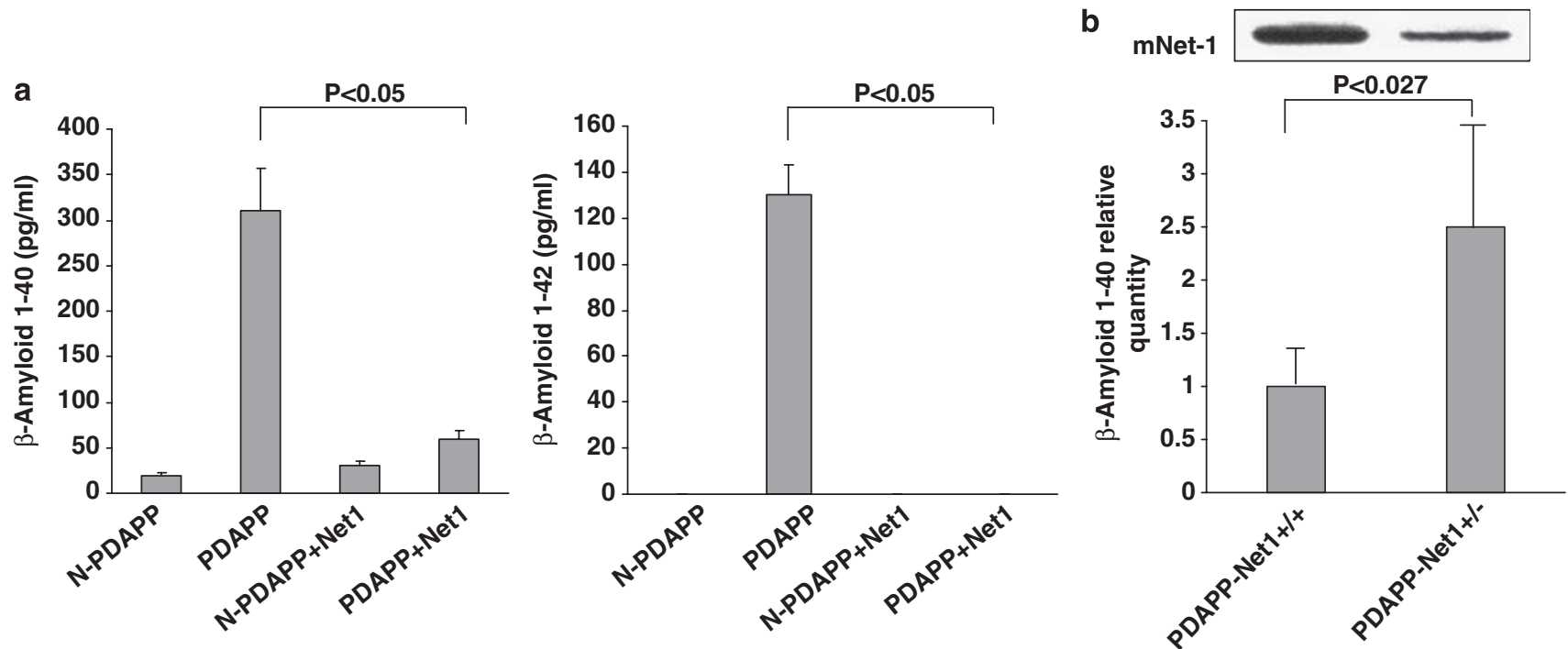

C

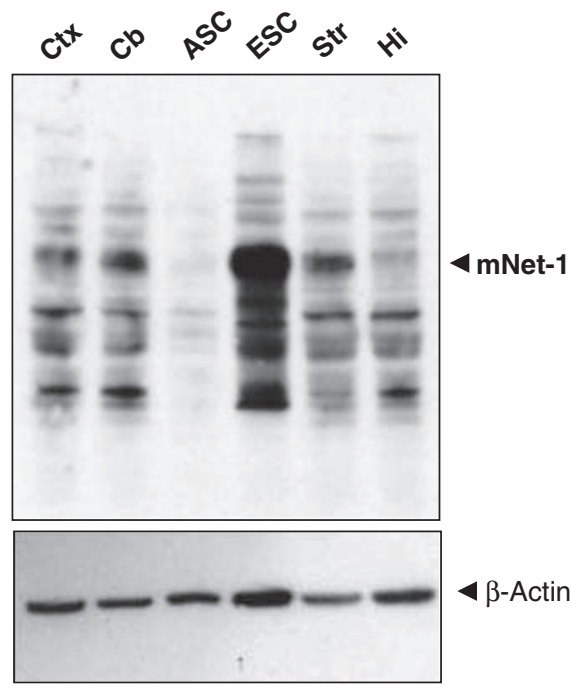

e

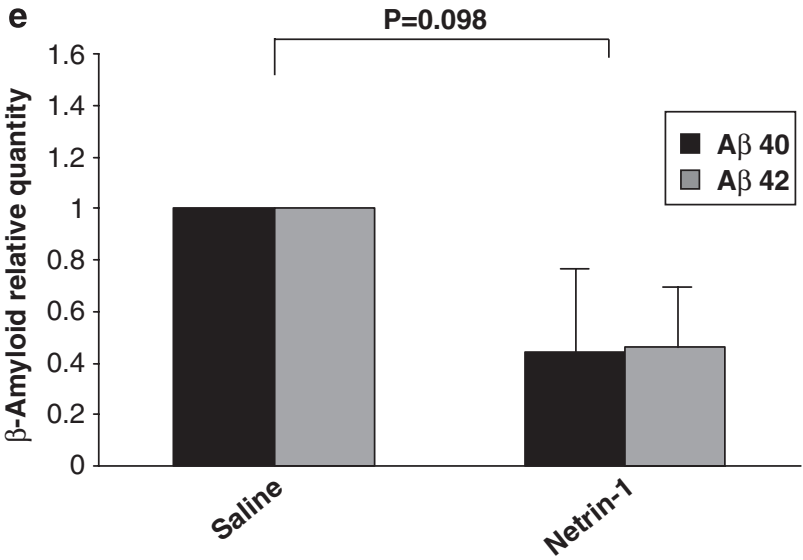

d

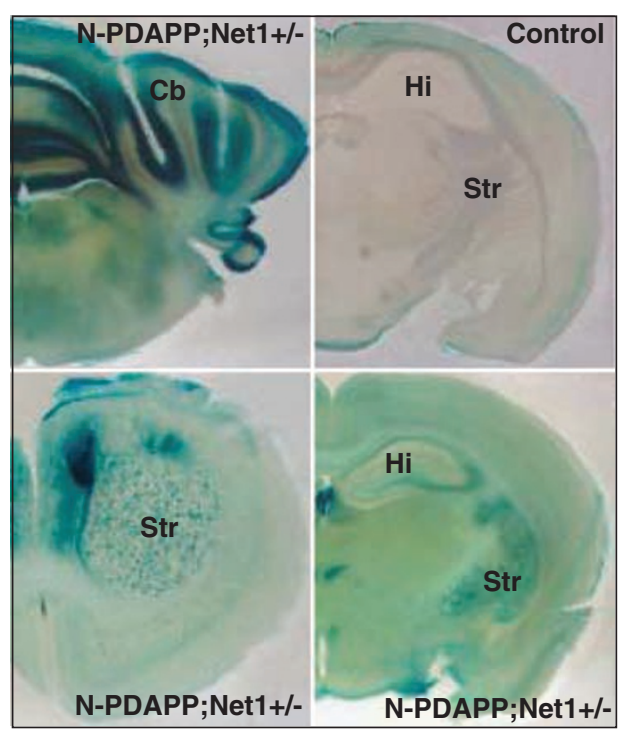

f

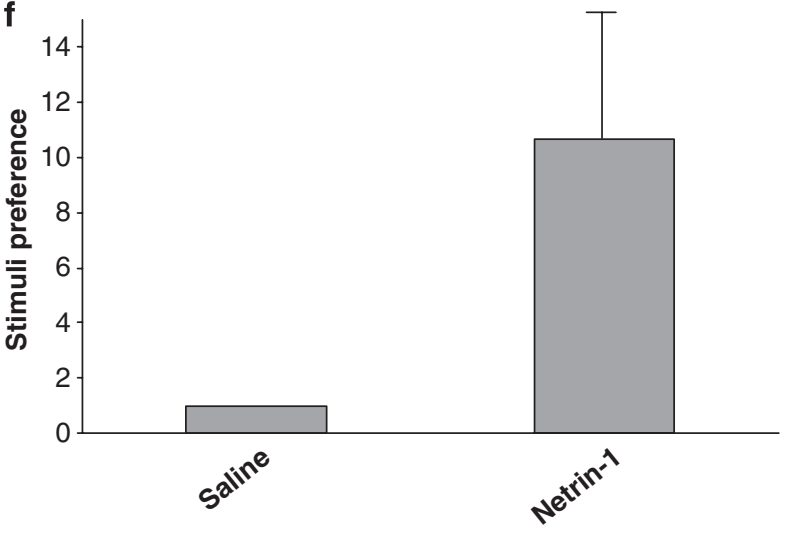


GST/netrin-1 was produced as described previously. ${ }^{28}$ His-netrin-1 was from R\&D systems (Minneapolis, MN, USA). bFGF was from ABcam. Recombinant APPs, $A \beta 1-42$ (and $A \beta 1-17$ ), and DCC-EC-FC were purchased from Sigma Aldrich, Anaspec, and R\&D systems, respectively.

Plasmid constructs. Full-length APP695 (pcDNA3-APP), netrin-1 (pGNET1myc), netrin-2 (pGNET2-myc), netrin G1 (pcDNA4-G1-netrin), domains V and VI of netrin-1 (pCEP4-netV-VI), APP-C100 (pclNeo-C100), APLP1 (pclNeo-APLP1), and DCC (pCMV-DCC-S)-expressing constructs were described previously. 8,10,29-32 APP613-695 was obtained by PCR using pcINeo-C100 as a PCR template and the following primers: $5^{\prime}$-CACCATGTTGGTGTTCTTTGCAGA-3' and $5^{\prime}$ - CTAGTT CTGCATCTGCTCAAA- $3^{\prime}$, and inserted into a pcDNA3.1TOPOd (Invitrogen). PG5E1B-luc (Gal4 reported construct), pMst (Gal4), pMst-APP (APP-Gal4), pMst$\mathrm{APP}^{*}$ (mutated form of APP unable to bind to Fe65) were described previously. ${ }^{17}$ TrkC (pCDNA3-TrkC-HA) was obtained by inserting into pCDNA3 vector, the rat TrkC coding sequence obtained from a pCMX-Trkc plasmid kindly provided by S Meakey.

APP/netrin-1 detection by ELISA assay. Here, 96-well plates (Immnunoplate Maxisorp, Nunc) were coated overnight at room temperature with a secreted form of APP (A $\beta$ and A $\beta 1-17$, respectively ) at a concentration of $2.5 \mu \mathrm{g} / \mathrm{ml}$ (respectively: $2.5,0.18,0.07 \mu \mathrm{g} / \mathrm{ml}$ ). After $1 \mathrm{~h}$ blocking at $37^{\circ} \mathrm{C}$ with $5 \%$ FCS in PBS, wells were washed (0.05\%Tween20 in PBS), followed by netrin-1 (Apotech) or bFGF incubation in a concentration range from $0.225 \mathrm{nM}-60 \mathrm{nM}$ at $37^{\circ} \mathrm{C}$ for $1 \mathrm{~h}$. Washed wells were incubated with an anti-FlagM2 (Sigma-Aldrich) or anti-bFGF antibody in blocking buffer for $30 \mathrm{~min}$ at $37^{\circ} \mathrm{C}$. An antimouse antibody coupled to HRP (Jackson ImmunoResearch Inc.) was added at a concentration of $0.8 \mu \mathrm{g} / \mathrm{ml}$ for $1 \mathrm{~h}$ at $37^{\circ} \mathrm{C}$. Colorimetric intensity was measured at a wavelength of $490 \mathrm{~nm}$ using a Victor station (Wallac).

Western blot and immunoprecipitation. Western blots were performed as described, ${ }^{12}$ using APP (C-terminal epitope:Sigma-Aldrich; N-terminal epitope: Sigma-Aldrich), A $\beta$ (biosource), Flag M2 (Sigma-Aldrich), DAB-1 (Exalpha Biologicals), Fe65 (Upstate), GST (Sigma-Aldrich),c-myc (Sigma-Aldrich), P75NGFR (abcam), TrkC (Santa-Cruz), or Net-1 antimouse monoclonal (R\&D systems) antibody. Coimmunoprecipitations from HEK293 and B103 were performed as described previously. ${ }^{33}$ Coimmunoprecipitations from primary culture were performed using the microbeads system developed by Miltenyi Biotech. Immunoprecipitation in developing brain was performed with a mixed mouse monoclonal anti-APP (5A3/ 1G7) antibody provided by E Koo. APP mutant mice were in a mixed background of C57BL6, 129SvEv, and 129 Ola and were described before. ${ }^{34}$ E14.5 mice embryos originating from the same cross APP $+/-$ XAPP $+/-$ were further dissected while genotyping was performed a posteriori.

Confocal analysis on primary cultures of neurons. Primary cultures of neurons were fixed in $4 \%$ PFA and stained with various primary antibodies ( $5 \mathrm{~A} 3 /$ $1 \mathrm{G} 7$, antinetrin 64 , or antiserum I (R1155)) followed by Alexa568-and/or Alexa488conjugated antimouse and/or antirabbit secondary antibodies, respectively. Stacks of images $(z$-step $=0.25 \mu \mathrm{m})$ were acquired with a laser scanning confocal microscope (Nikon PCM-2000) using a 100X objective and a 2.7 digital zoom, collected using SimplePCl software (Compix Inc., Lake Oswego, OR, USA) and processed in an SGI Octane R12 computer running Bitplane's Advanced Imaging Software suite.

Organotypic culture and $\mathbf{A} \boldsymbol{\beta}$ release determination. PDAPP(J20) mice were described before. ${ }^{27}$ In all, $250 \mu \mathrm{m}$ coronal brain slices were cut from P1 transgenic and nontransgenic littermates' whole brain to establish organotypic cultures. Tissues were incubated in $0.5 \% \mathrm{D}$-glucose, $25 \%$ fetal bovine serum, $25 \%$ Hank's buffered saline solution, and in Opti-MEM (Invitrogen). Recombinant netrin-1, NGF, or IGF-1 was added to the media immediately after plating and every $24 \mathrm{~h}$. After 3 days, $A \beta 1-40$ and $A \beta 1-42$ were quantitated in the culture media using specific ELISA assays (Biosource, Camarillo, CA, USA). To measure A $\beta$ in a context of lower netrin-1 concentration, transgenic PDAPP(J20) mice were crossed with netrin-1 +/ - mice. Netrin-1 mutant mice were described before. ${ }^{9,35}$ Mice with adequate genotypes (PDAPP/netrin-1 $+/$ - and PDAPP/netrin-1 $+/+$ ) were analyzed for $A \beta$ level using the specific above ELISA assay.

Intracerebroventricular pump injection and behavioral testing in PDAPP mice. Recombinant mouse netrin-1 (R\&D) was infused in the left ventricle using Alzet osmotic pumps (Alzet 1002, $0.25 \mu \mathrm{l} / \mathrm{h}$ release rate) for 12-13 days. To assess memory, the simple ON test was used essentially as described ${ }^{27}$ and in accordance with the ON SOP from the Murine Behavioral Assessment Laboratory at the University of California, Davis, CA, USA) 1 day before euthanasia. Preference for novelty in the $\mathrm{ON}$ test depends on the ability of mice to recall prior experience when presented with a previously encountered scent together with a novel one. Percentage of total time spent investigating the novel scent (preference) was scored and used as a measure of memory of previous experience.

Acknowledgements. We thank E Koo, V Castellani for discussion, H Arakawa for the GST/netrin-1-expressing construct and T Südhof for the APPGal4 transactivation system. We thank C Guix, MM Coissieux, A Tang, O Gorostiza and $D$ Crippen for excellent technical assistance and $C$ Ségura-Ferlay for statistical analysis. This work was supported by the Ligue Contre le Cancer (PM), the fondation pour le Cerveau (PM), the ARC (PM), the ANR (PM), the NIH (NS33376 to $\mathrm{PM}$ and $\mathrm{DEB})$, the Joseph Drown Foundation (DEB), the John Douglas French Foundation (VG), and the Alzheimer's Association (VG). VG thanks Mrs Eloise Goodhew Barnett for her support. FC is supported by a fellowship from the Portuguese Science and Technology Foundation (POCl2010).

\section{Conflict of interest. Authors declare to have no conflict of interest.}

1. Hansen LA, Terry RD. Position paper on diagnostic criteria for Alzheimer disease. Neurobiol Aging 1997; 18 (4 Suppl): S71-S73.

2. Koo EH. The beta-amyloid precursor protein (APP) and Alzheimer's disease: does the tail wag the dog? Traffic 2002; 3: 763-770.

3. Turner PR, O' Connor K, Tate WP, Abraham WC. Roles of amyloid precursor protein and its fragments in regulating neural activity, plasticity and memory. Prog Neurobiol 2003; 70: $1-32$.

4. Beher D, Hesse L, Masters CL, Multhaup G. Regulation of amyloid protein precursor (APP) binding to collagen and mapping of the binding sites on APP and collagen type I. J Biol Chem 1996; 271: 1613-1620

5. Caceres J, Brandan E. Interaction between Alzheimer's disease beta A4 precursor protein (APP) and the extracellular matrix: evidence for the participation of heparan sulfate proteoglycans. J Cell Biochem 1997; 65: 145-158.

6. Williamson TG, Mok SS, Henry A, Cappai R, Lander AD, Nurcombe V et al. Secreted glypican binds to the amyloid precursor protein of Alzheimer's disease (APP) and inhibits APP-induced neurite outgrowth. J Biol Chem 1996; 271: 31215-31221.

7. Ma QH, Futagawa T, Yang WL, Jiang XD, Zeng L, Takeda $Y$ et al. A TAG1-APP signalling pathway through Fe65 negatively modulates neurogenesis. Nat Cell Biol 2008; 10: 283-294.

8. Serafini T, Kennedy TE, Galko MJ, Mirzayan C, Jessell TM, Tessier-Lavigne M. The netrins define a family of axon outgrowth-promoting proteins homologous to C. elegans UNC-6. Cell 1994; 78: 409-424.

9. Serafini T, Colamarino SA, Leonardo ED, Wang H, Beddington R, Skarnes WC et al. Netrin-1 is required for commissural axon guidance in the developing vertebrate nervous system. Cell 1996; 87: 1001-1014.

10. Keino-Masu K, Masu M, Hinck L, Leonardo ED, Chan SS, Culotti JG et al. Deleted in colorectal cancer (DCC) encodes a netrin receptor. Cell 1996; 87: 175-185.

11. Mazelin L, Bernet A, Bonod-Bidaud C, Pays L, Arnaud S, Gespach C et al. Netrin-1 controls colorectal tumorigenesis by regulating apoptosis. Nature 2004; 431: 80-84.

12. Mehlen P, Rabizadeh S, Snipas SJ, Assa-Munt N, Salvesen GS, Bredesen DE. The DCC gene product induces apoptosis by a mechanism requiring receptor proteolysis. Nature 1998; 395: 801-804

13. Small DH, Nurcombe V, Reed G, Clarris H, Moir R, Beyreuther K et al. A heparin-binding domain in the amyloid protein precursor of Alzheimer's disease is involved in the regulation of neurite outgrowth. J Neurosci 1994; 14: 2117-2127.

14. Lu X, Le Noble F, Yuan L, Jiang Q, De Lafarge B, Sugiyama D et al. The netrin receptor UNC5B mediates guidance events controlling morphogenesis of the vascular system. Nature 2004; 432: 179-186.

15. Trommsdorff M, Borg JP, Margolis B, Herz J. Interaction of cytosolic adaptor proteins with neuronal apolipoprotein E receptors and the amyloid precursor protein. J Biol Chem 1998; 273: 33556-33560.

16. Ando K, lijima KI, Elliott Jl, Kirino Y, Suzuki T. Phosphorylation-dependent regulation of the interaction of amyloid precursor protein with $\mathrm{Fe} 65$ affects the production of beta-amyloid. J Biol Chem 2001; 276: 40353-40361.

17. Cao X, Sudhof TC. A transcriptionally [correction of transcriptively] active complex of APP with Fe65 and histone acetyltransferase Tip60. Science 2001; 293: 115-120.

18. Kimberly WT, Zheng JB, Guenette SY, Selkoe DJ. The intracellular domain of the betaamyloid precursor protein is stabilized by Fe65 and translocates to the nucleus in a notchlike manner. J Biol Chem 2001; 276: 40288-40292.

19. Cao X, Sudhof TC. Dissection of amyloid-beta precursor protein-dependent transcriptiona transactivation. J Biol Chem 2004; 279: 24601-24611. 
20. Kimberly WT, Zheng JB, Town T, Flavell RA, Selkoe DJ. Physiological regulation of the beta-amyloid precursor protein signaling domain by c-Jun N-terminal kinase JNK3 during neuronal differentiation. J Neurosci 2005; 25: 5533-5543.

21. Mucke L, Masliah E, Yu GQ, Mallory M, Rockenstein EM, Tatsuno G et al. High-level neuronal expression of abeta 1-42 in wild-type human amyloid protein precursor transgenic mice: synaptotoxicity without plaque formation. $J$ Neurosci 2000; 20: 4050-4058.

22. von Rotz RC, Kohli BM, Bosset J, Meier M, Suzuki T, Nitsch RM et al. The APP intracellular domain forms nuclear multiprotein complexes and regulates the transcription of its own precursor. J Cell Sci 2004; 117 (Pt 19): 4435-4448.

23. Livesey FJ, Hunt SP. Netrin and netrin receptor expression in the embryonic mammalian nervous system suggests roles in retinal, striatal, nigral, and cerebellar development. Mol Cell Neurosci 1997; 8: 417-429

24. Colicos MA thesis. McGill University 1999.

25. Llambi $F$, Causeret $F$, Bloch-Gallego $E$, Mehlen $P$. Netrin-1 acts as a survival factor via its receptors UNC5 H and DCC. EMBO J 2001; 20: 2715-2722.

26. Hsiao KK, Borchelt DR, Olson K, Johannsdottir R, Kitt C, Yunis W et al. Age-related CNS disorder and early death in transgenic FVB/N mice overexpressing Alzheimer amyloid precursor proteins. Neuron 1995; 15: 1203-1218.

27. Galvan V, Gorostiza OF, Banwait S, Ataie M, Logvinova AV, Sitaraman S et al. Reversal of Alzheimer's-like pathology and behavior in human APP transgenic mice by mutation of Asp664. Proc Natl Acad Sci USA 2006; 103: 7130-7135.

28. Tanikawa C, Matsuda K, Fukuda S, Nakamura $Y$, Arakawa H. p53RDL1 regulates p53-dependent apoptosis. Nat Cell Biol 2003; 5: 216-223.
29. Lu DC, Rabizadeh S, Chandra S, Shayya RF, Ellerby LM, Ye X et al. A second cytotoxic proteolytic peptide derived from amyloid beta-protein precursor. Nat Med 2000; 6: 397-404.

30. Lu DC, Shaked GM, Masliah E, Bredesen DE, Koo EH. Amyloid beta protein toxicity mediated by the formation of amyloid-beta protein precursor complexes. Ann Neurol 2003; 54: 781-789.

31. Galvan V, Chen S, Lu D, Logvinova A, Goldsmith P, Koo EH et al. Caspase cleavage of members of the amyloid precursor family of proteins. J Neurochem 2002; 82: 283-294.

32. Homayouni R, Rice DS, Sheldon M, Curran T. Disabled-1 binds to the cytoplasmic domain of amyloid precursor-like protein 1. J Neurosci 1999; 19: 7507-7515.

33. Forcet C, Ye X, Granger L, Corset V, Shin H, Bredesen DE et al. The dependence receptor DCC (deleted in colorectal cancer) defines an alternative mechanism for caspase activation. Proc Natl Acad Sci USA 2001; 98: 3416-3421.

34. Heber S, Herms J, Gajic V, Hainfellner J, Aguzzi A, Rulicke T et al. Mice with combined gene knock-outs reveal essential and partially redundant functions of amyloid precursor protein family members. J Neurosci 2000; 20: 7951-7963.

35. Forcet C, Stein E, Pays L, Corset V, Llambi F, Tessier-Lavigne M et al. Netrin-1-mediated axon outgrowth requires deleted in colorectal cancer-dependent MAPK activation. Nature 2002; 417: 443-447.

36. Costes SV, Daelemans D, Cho EH, Dobbin Z, Pavlakis G, Lockett S. Automatic and quantitative measurement of protein-protein colocalization in live cells. Biophys J 2004; 86: 3993-4003.

37. Selkoe DJ, Podlisny MB, Joachim CL, Vickers EA, Lee G, Fritz LC et al. Beta-amyloid precursor protein of Alzheimer disease occurs as 110- to 135-kilodalton membrane-associated proteins in neural and nonneural tissues. Proc Natl Acad Sci USA 1988; 85: 7341-7345.

\section{Supplementary Information accompanies the paper on Cell Death and Differentiation website (http://www.nature.com/cdd)}

